\title{
Self-taught hashing using deep graph embedding for large-scale image retrieval (retraction notice)
}

\author{
Ruiling Zhou, Jinguo Zhao, Rui He, and Xinyu Zhang \\ Hunan Institute of Technology, School of Computer and Information Science, \\ Hengyang, China
}

J. of Electronic Imaging, 29(3), 033014 (2020). https://doi.org/10.1117/1.JEl.29.3.033014

Online Publication Date: 19 June 2020

Withdrawn from Publication: 03 September 2020.

This paper was retracted from the SPIE Digital Library on September 3, 2020, at the request of the authors, who found several serious errors in the experimental results due to incorrect previous experimental setup. 